\title{
The Helioseismic and Magnetic Imager (HMI) Investigation for the Solar Dynamics Observatory (SDO)
}

\author{
P.H. Scherrer • J. Schou • R.I. Bush • A.G. Kosovichev • R.S. Bogart • J.T. Hoeksema • \\ Y. Liu • T.L. Duvall Jr. · J. Zhao • A.M. Title • C.J. Schrijver · T.D. Tarbell • \\ S. Tomczyk
}

Received: 6 June 2011 / Accepted: 4 August 2011 / Published online: 18 October 2011

(C) The Author(s) 2011. This article is published with open access at Springerlink.com

\begin{abstract}
The Helioseismic and Magnetic Imager (HMI) instrument and investigation as a part of the NASA Solar Dynamics Observatory (SDO) is designed to study convection-zone dynamics and the solar dynamo, the origin and evolution of sunspots, active regions, and complexes of activity, the sources and drivers of solar magnetic activity and disturbances, links between the internal processes and dynamics of the corona and heliosphere, and precursors of solar disturbances for space-weather forecasts. A brief overview of the instrument, investigation objectives, and standard data products is presented.
\end{abstract}

Keywords Solar Dynamics Observatory · Helioseismology · Instrumentation and data management $\cdot$ Magnetic fields, photosphere

\section{Overview}

The Helioseismic and Magnetic Imager (HMI) investigation is part of the NASA Solar Dynamics Observatory (SDO) mission, which is the first flight component of the NASA

The solar Dynamics Observatory

Guest Editors: W. Dean Pesnell, Phillip C. Chamberlin, and Barbara J. Thompson

P.H. Scherrer (凶) · J. Schou · R.I. Bush · A.G. Kosovichev · R.S. Bogart · J.T. Hoeksema · Y. Liu •

J. Zhao

W.W. Hansen Experimental Physics Laboratory, Stanford University, Stanford, CA 94305-4085, USA

e-mail: pscherrer@solar.stanford.edu

url: http://hmi.stanford.edu

T.L. Duvall Jr.

Laboratory for Astronomy and Solar Physics, NASA Goddard Space Flight Center, Greenbelt, MD 20771, USA

A.M. Title · C.J. Schrijver · T.D. Tarbell

Lockheed Martin Solar and Astrophysics Laboratory, 3251 Hanover St., Palo Alto, CA 94304, USA

S. Tomczyk

High Altitude Observatory, National Center for Atmospheric Research, 3080 Center Green CG-1,

Boulder, CO 80301, USA 
Living With a Star (LWS) program. HMI is the name of one of the instruments on SDO and is also the name of a science investigation. This article provides a terse overview of both aspects of HMI primarily as an introduction to several more detailed articles. HMI is a joint project of the Stanford University Hansen Experimental Physics Laboratory, the Lockheed Martin Solar and Astrophysics Laboratory, the High Altitude Observatory, and Co-Investigators at 21 additional institutions.

\subsection{Top Level Goals}

The overarching purpose of SDO is to make progress in the capability to understand and predict solar events that contribute to variability in the Earth's space environment. The solar variability that generates events important for Earth is driven by emergence and evolution of solar magnetic active regions. The primary goal of the HMI investigation is to study the origin of solar variability and to characterize and understand the Sun's interior and the various components of magnetic activity. These top-level objectives are described in the LWS Science Architecture Team Report (Mason and LWS Panel, 2001) and the SDO Science Definition Team Report (Hathaway and SDO SDT Panel, 2001).

The range of science investigations that HMI can contribute to, as understood at the time of the proposal to NASA for the HMI investigation, was described in that proposal (Scherrer and HMI Team, 2002). The expectation is that only some of the scientific research identified in the proposal will be pursued by the core HMI team at Stanford University with many of the studies to be conducted by the Co-Investigator team and by many others who are enticed by the challenging topics enabled by the availability of the HMI data collection. Therefore this article should not be construed as the list of investigations that will be pursued by the HMI team but rather as the list of topics that were examined in some detail to ensure that the observational and pipeline processing capabilities of the HMI project can make significant contributions to their better understanding. A detailed discussion of the initial investigation expectations was provided to NASA as a required document and simultaneously to the community via web availability in November 2004 in the "HMI Science Plan" (Kosovichev and HMI Science Team, 2004). Since this expanded description as well as the original more condensed version in the HMI 2002 proposal have been on the web for several years, the anticipated science investigation topics need not be fully detailed here. While these defining documents anticipated the SDO launch to be in 2006 or 2007 to begin a five-year base mission at a time of low solar activity, the Sun managed to delay the start of the current cycle until the SDO actual launch in early 2010. The earlier documents' description of the science objectives of HMI are still valid and are therefore the primary basis for this article.

The specific scientific objectives of the HMI investigation are to measure and study: convection-zone dynamics and the solar dynamo; the origin and evolution of sunspots, active regions and complexes of activity; the sources and drivers of solar magnetic activity and disturbances; links between the internal processes and dynamics of the corona and heliosphere; and precursors of solar disturbances for space-weather forecasts. These objectives will be described in some more detail below.

\subsection{Scope}

This article is one of several that describe the HMI investigation, instrument, and processing plans as of roughly the time of the SDO launch. It covers primarily an overview of the planned science investigation with only a very terse mention of the other aspects, which are described in some detail in the associated articles. The detailed description of the HMI instrument is contained in Schou et al. (2011) and it should be cited by users of data produced 
by HMI. Some of the aspects of the instrument and data-analysis pipeline required specific HMI developments and have been detailed in other associated articles. These include detailed discussions of the HMI image quality (Wachter et al., 2011), the HMI filter characteristics (Couvidat et al., 2011), the instrumental polarization calibration (Schou et al., 2010), the vector-field inversion technique (Borrero et al., 2010), and the time-distance helioseismology travel-time measurement methods (Couvidat et al., 2010), and the time-distance helioseismology processing pipeline (Zhao et al., 2011). The expectation is that there will be additional articles describing the actual on-orbit characteristics of data products. These actual data performance articles will not be available until more than a year into the mission. Data-product descriptions and performance notes will be updated and maintained via links to the web at http://hmi.stanford.edu and http://jsoc.stanford.edu for the duration of the SDO mission.

\subsection{The Helioseismic and Magnetic Imager Instrument}

To support these objectives, the HMI instrument has been designed to produce measurements in the form of filtergrams in a set of polarizations and spectral-line positions at a regular cadence for the duration of the mission. The HMI instrument makes measurements of the motion of the solar photosphere to study solar oscillations and measurements of the polarization in a spectral line to study all components of the photospheric magnetic field. The HMI instrument is an enhanced version of the Michelson Doppler Imager (MDI) instrument which is part of the Solar and Heliospheric Observatory (SOHO: Scherrer et al., 1995). HMI has significant heritage from MDI with modifications to allow higher resolution, higher cadence, and the addition of a second camera to provide additional polarization measurements without interfering with the filtergram cadence needed for helioseismology. HMI provides stabilized one-arcsecond resolution full-disk Doppler velocity, line-of-sight magnetic flux, and continuum proxy images every 45 seconds, and vector magnetic field maps every 90 or 135 seconds depending on the image frame sequence selected. The solar image nearly fills the $4096 \times 4096$ pixel CCD camera allowing 0.5 arcsecond per pixel. The HMI instrument was developed at the Lockheed Martin Solar and Astrophysics Laboratory (LMSAL) in collaboration with Stanford University as part of the Stanford Lockheed Institute for Space Research collaboration.

\subsection{HMI Data Products}

The significant data stream to be provided by HMI must be analyzed and interpreted with advanced tools that permit study of the complex flows and structures deduced from helioseismic inversion together with direct measurements of surface magnetic fields. It is essential to have convenient access to all data products - Dopplergrams, full-disk vector magnetograms, sub-surface flow-field and sound-speed maps, as well as coronal magnetic-field estimates - for any region or event selected for analysis. The other SDO investigation that generates a large dataflow, the Atmospheric Imaging Assembly (AIA: Lemen et al., 2011) developed and operated by LMSAL, and HMI have combined operations and data processing capabilities in the form of the HMI/AIA Joint Science Operations Center (JSOC). The JSOC consists of three components: the JSOC Instrument Operations Center (IOC) located at LMSAL, the Science Data Processing facility (SDP) located at Stanford University, and the AIA Visualization Center (AVC) located at LMSAL. The JSOC-SDP provides a data system for archiving the HMI and AIA data and derived data products with convenient access to the data by all interested investigators as well as the general public. JSOC-managed data 
products are available directly to the user via web tools, with key products also distributed through the Virtual Solar Observatory (VSO). The SDP facility also provides processing capabilities for higher-level HMI data products. Sufficient computing capability is in place to allow the investigation of a subset of the HMI science objectives.

HMI will obtain filtergrams in various positions in the Fe I $617.3 \mathrm{~nm}$ spectral line and a set of polarizations at a regular cadence for the duration of the mission. Several higher levels of data products will be produced from the filtergrams. The basic science observables are full-disk Doppler velocity, a continuum brightness proxy, line-of-sight magnetic flux, and vector magnetic field. These are available at full spatial resolution at a 45-second cadence. Additional estimates are generated for the line width and line depth. The basic vector magnetic-field quantity is an array of 24 filtergrams (six wavelengths, four Stokes parameters) averaged over 12 minutes. Additionally, the results of Milne-Eddington inversions and the proper disambiguated field vector will be provided. Derived data products sampled and averaged at various resolutions and cadence and sub-image samples tracked with solar rotation are also available. A selection of these will be made available on a regular basis, and other data products will be made on request. Also of great potential value are derived products such as sub-surface flow maps, far-side activity maps, and coronal and solar-wind models which require longer sequences of observations. A selection of these will also be produced in the processing pipeline in near-real time. Most of the HMI Co-Investigators have specific roles in providing software to enable production of the various standard data products. The processing of HMI data is accomplished in a "pipeline" moving data from the observed filtergrams through multiple steps to basic science products (the "observables") and higher-level derived products such as sub-photospheric flow maps. The basic processing pipeline is shown in Figure 1. The JSOC-SDP is described in more detail on its web page at http://jsoc.stanford.edu.

\subsection{Team, Collaborations and Community}

The HMI investigation plan identifies a broad range of scientific objectives that can be addressed with HMI observations. HMI provides a unique set of data required for scientific understanding, detailed characterization, and advanced warning of the effects of solar disturbances on global changes, space weather, human exploration and development, and technological systems. HMI also provides correlative data required for accomplishing objectives of the other SDO instruments. A significant research effort must be undertaken to exploit the capabilities of HMI and SDO.

The HMI program itself does not have sufficient resources to pursue all of the science goals possible with HMI data or even all of the goals identified in this article; neither was it planned to. The expectation is that Co-Investigators as well as many other interested investigators will find support as needed to carry out the studies that will in the end make most use of HMI data to achieve the goals of HMI, SDO, and LWS. To help this process, NASA has selected a consortium led by D. Braun at Colorado Research Associates (a division of Northwest Research Associates) to support development of next-generation helioseismology analysis tools. Tools developed by this supporting project will be available in several years. Most HMI analysis is likely to be carried out with support from NASA LWS and Guest Investigator grant programs in the US and from other national science-support agencies in other countries.

To further the goals of nationally funded research it is important that the science teams pursue careful science investigations and tell others about the results - and not just the science community. SDO investigations and HMI in particular have aspects which will be of 


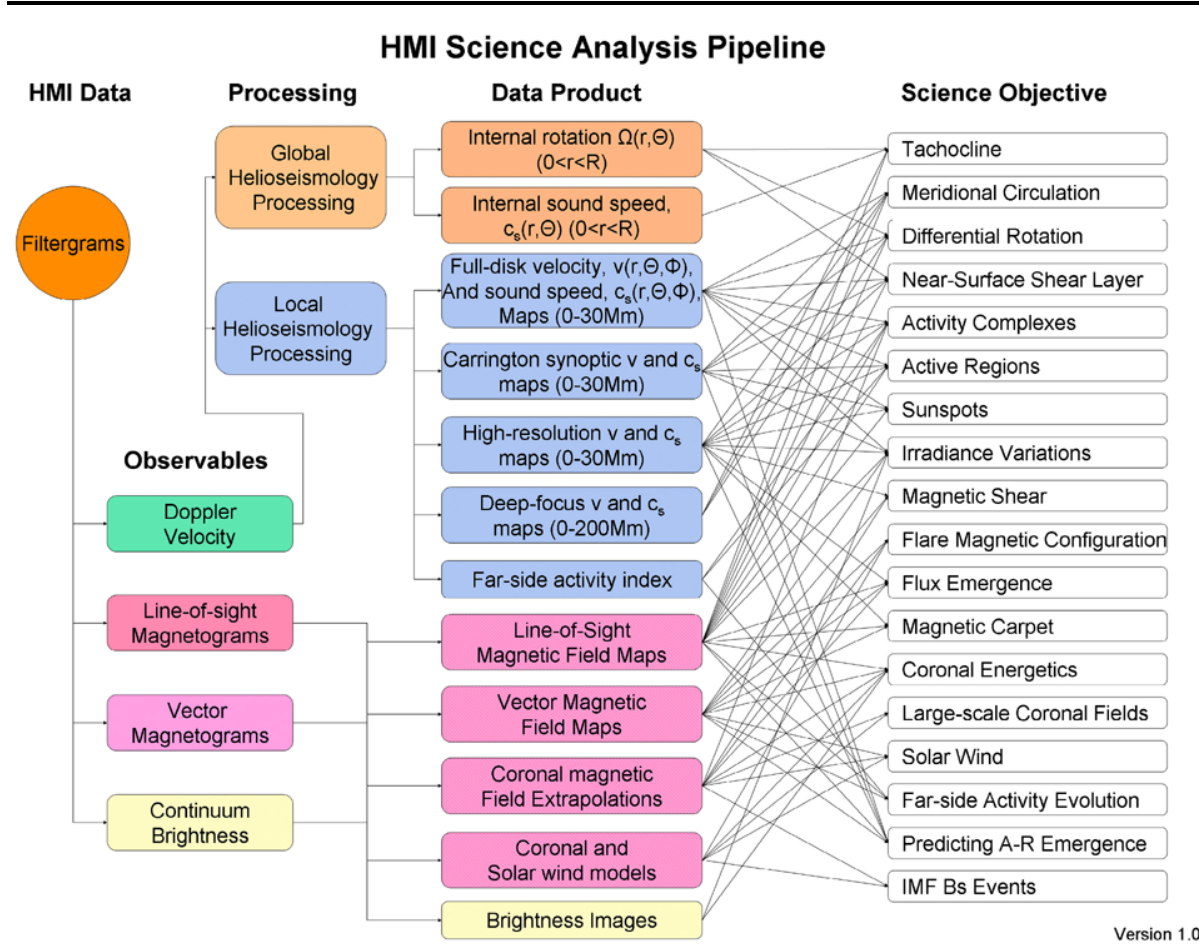

Figure 1 Schematic flow diagram for HMI data-analysis pipeline. The observed filtergrams are used primarily to produce "observables" which are the level-1 data products available to the community. Further higher-level products such as internal rotation and coronal-field extrapolations are produced on a regular cadence and are expected to be the data used for science investigations, shown by the arrows, to achieve the HMI science objectives.

interest to the public at large. Also SDO and HMI will offer excellent opportunities for developing interesting and timely educational material. A highly leveraged, collaborative Education and Public Outreach (E/PO) program is a key part of this investigation (Drobnes et al., 2011). The EPO program is described in components of the http://solar-center.stanford.edu access point.

\subsection{History}

In January 1996 when the first SOHO/MDI test images became available, Duvall, Kosovichev, and colleagues (Duvall et al., 1993, 1997; Kosovichev, 1996) began an investigation to explore using the newly developed time-distance method of local-area helioseismology with MDI high-resolution data. This method proved to be immediately useful but constrained by the limited field of view of the MDI high-resolution (1.2 arcsec) configuration. The success of the method, limited field of view, and limited telemetry opportunities suggested that some future mission would be needed to fully exploit the opportunity to make detailed probes in the near-surface solar interior. This conclusion was presented at the NASA Roadmap conferences at JHU-APL in April 1996 and JPL in October 1996. In 1998 a proposal was submitted to NASA for a MIDEX mission named "Hale" which included a fulldisk high-resolution MDI-like instrument and an EUV imaging instrument. Figure 2, which shows wave speed beneath a sunspot, was made for that proposal with data obtained with 


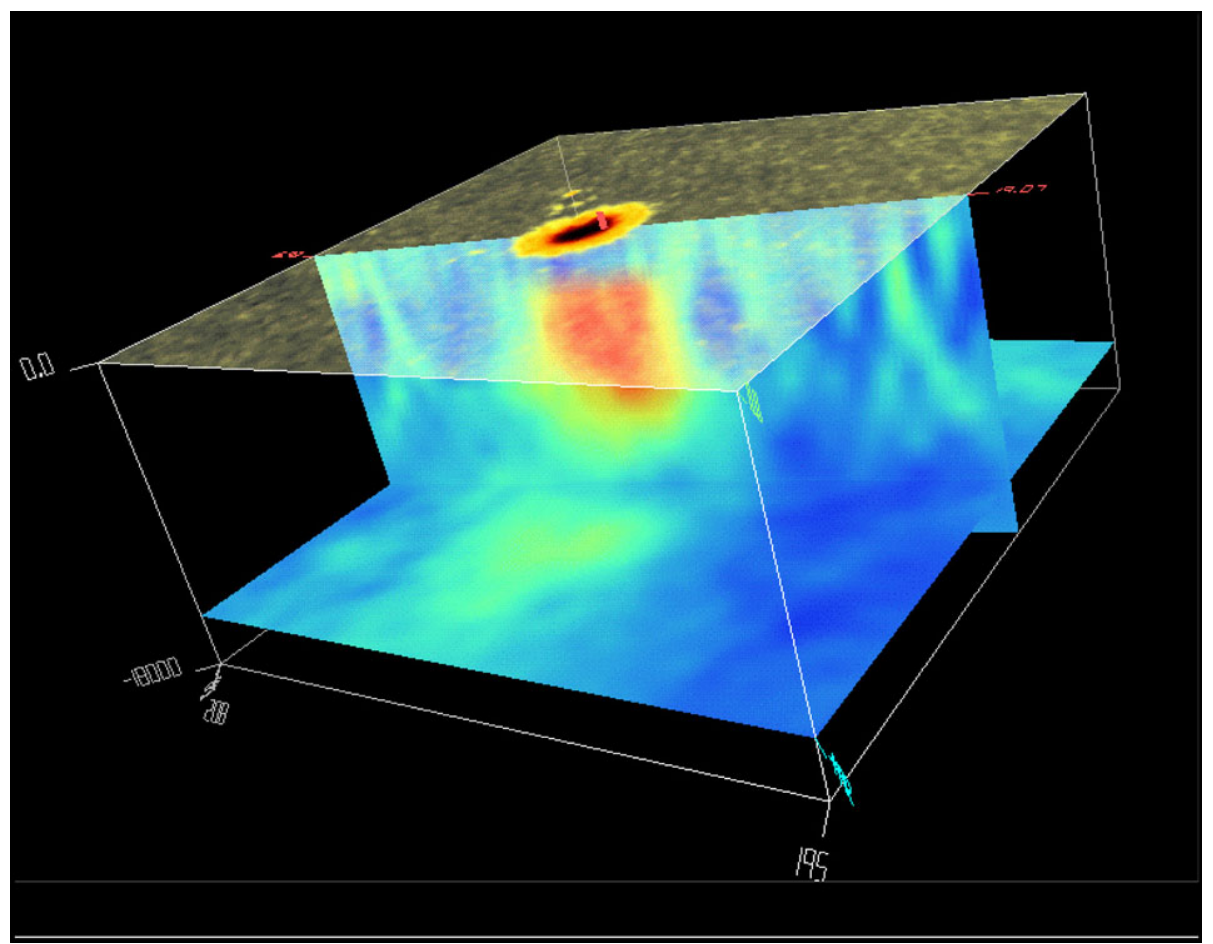

Figure 2 Helioseismic tomography used to map wave speed beneath the sunspot of NOAA AR 8243 on 18 June 1998 computed with the method developed by Duvall et al. (1997). The top surface is the MDI continuum-intensity proxy and interior slices are wave speed with blue tones for wave speed slower than average for the box and red colors for higher speed. The box is about $195 \mathrm{Mm}$ square and $18 \mathrm{Mm}$ deep. Analysis method was described by Kosovichev, Duvall, and Scherrer (2000) and the figure is from a proposal to the NASA 1998 MIDEX opportunity - Hale: Exploring Solar Activity.

SOHO/MDI in June 1998. This was the first sunspot that traversed the MDI high-resolution field while high-rate telemetry was available and was in the week before the 1998 SOHO mission disruption. The MIDEX proposal was not selected but the concept was included in the Sun-Earth Connections Roadmap of 2000 as a mission named SONAR which contained the essence of the eventual SDO. Over the next two years these concepts helped to form the SDO mission concept as part of the then new Living With a Star program. Broader community input into the SDO Definition Study helped to define a "strawman" HMI instrument which led to the full HMI proposal for the SDO mission in April 2002. About fourteen years elapsed between the first suggestion of a mission with HMI capabilities and the beginning of the science phase of SDO in May 2010.

\section{HMI Science Goals}

The HMI investigation encompasses three primary LWS objectives: first, to determine how and why the Sun varies; second, to improve our understanding of how the Sun drives global change and space weather; and third, to determine to what extent predictions of space weather and global change can be made and to prototype predictive techniques. 


\subsection{Science Overview}

The Sun is a magnetic star. High-speed solar wind, the structure of heliospheric current sheets, coronal holes, coronal mass ejections, flares, and variable components of irradiance are all linked to the variability of magnetic fields that pervade the solar interior and atmosphere. Many of these events can have profound impacts on our technological society, so understanding them is a key objective for LWS. A central question is the origin of solar magnetic fields. Most striking is that the Sun exhibits 22-year cycles of global magnetic activity involving magnetic active regions with very well-defined polarity rules producing global-scale magnetic patterns. Coexisting with these large-scale magnetic structures and concentrated active regions are ephemeral active regions and other compact and intense flux structures (Zwaan, 1987) that emerge randomly over much of the solar surface forming a "magnetic carpet" (Title and Schrijver, 1998).

The extension of these changing fields, at all scales, into the solar atmosphere creates coronal activity, which is the source of space-weather variability. The HMI scientific investigation addresses the fundamental problems of solar variability with studies in all interlinked temporal and spatial domains, including global scale, active regions, small scale, and coronal connections. One of the prime objectives of the Living With a Star program is to understand how well predictions of evolving space-weather variability can be made. The HMI investigation will examine these questions in parallel with the fundamental science questions of how the Sun varies and how that variability drives global change and space weather.

The tools that will be used in the HMI program include: helioseismology to map and probe the solar convection zone where a magnetic dynamo likely generates this diverse range of activity; measurements of the photospheric magnetic field, which results from the internal processes and drives the processes in the atmosphere; and brightness measurements, which can reveal the relationship between magnetic and convective processes and solar irradiance variability.

Helioseismology, which uses solar oscillations to probe flows and structures in the solar interior, is providing remarkable new perspectives about the complex interactions between highly turbulent convection, rotation, and magnetism. It has revealed a region of intense rotational shear at the base of the convection zone (Schou et al., 1998), called the tachocline, which is the likely seat of the global dynamo. Convective flows also have a crucial role in advecting and shearing the magnetic fields, twisting the emerging flux tubes and displacing the photospheric footpoints of magnetic structures present in the corona. Flows at all spatial scales influence the evolution of the magnetic fields, including how the fields generated near the base of the convection zone rise and emerge at the solar surface, and how the magnetic fields already present at the surface are advected and redistributed. Both of these mechanisms contribute to the establishment of magnetic-field configurations that may become unstable and lead to eruptions that affect the near-Earth environment (e.g., van Driel-Gesztelyi and Culhane, 2009).

Local-area helioseismology methods have begun to reveal the great complexity of rapidly evolving 3D magnetic structures and flows in the sub-surface shear layer in which the sunspots and active regions are embedded. Most of these techniques were developed by members of the extended HMI team for analysis of MDI and GONG observations. As useful as they are, the limitations of MDI telemetry and the limited field of view at high resolution and limited GONG resolution have prevented the full exploitation of the methods to answer important questions about the origins of solar variability. By using these techniques on continuous, full-disk, high-resolution observations, HMI will enable detailed probing of dynamics and magnetism within the near-surface shear layer, and provide sensitive measures of variations in the tachocline. 
Just as existing helioseismology experiments have shown that new techniques can lead to new understanding; methods to measure the full vector magnetic field have been developed and shown the potential for significantly enhanced understanding of magnetic evolution and connections. What existing and planned ground-based programs cannot do, and what Hinode cannot do, is to observe the full-disk vector magnetic field continuously at a cadence sufficient to follow the development of activity. The HMI vector magnetic-field measurement capability, in combination with the other SDO instruments and other programs (e.g., STEREO, Hinode, and SOLIS) will provide data important for connecting solar variability in the solar interior to variability in the solar atmosphere, and to the propagation of solar variability in the heliosphere.

HMI brightness observations will provide information about the areal distribution of magnetic and convective contributions to irradiance variations, and also about variations of the solar radius and shape.

\subsection{Scientific Goals}

The broad goals described above will be addressed in a coordinated investigation of a number of parallel studies. These segments of the HMI investigation listed in Section 1.1 are described in some more detail here.

The goals represent long-standing problems that can be addressed by a number of more immediate tasks. The description of these tasks reflects our current level of understanding and will obviously evolve in the course of the investigation. Some of the currently mostimportant tasks are described below. The five broad objectives above are themselves described as sets of component objectives. Figure 1 shows a conceptual flow diagram connecting HMI planned data products with these component objectives.

\subsubsection{Convection-Zone Dynamics and the Solar Dynamo}

Fluid motions inside the Sun are believed to generate solar magnetic fields. Complex interactions between turbulent convection, rotation, large-scale flows, and magnetic field produce regular patterns of solar activity changing quasi-periodically with the solar cycle. How are variations in the solar cycle related to the internal flows and surface magnetic field? How is the differential rotation produced? What is the structure of the meridional flow and how does it vary? What roles do the zonal-flow pattern and the variations of the rotation rate in the tachocline play in the solar dynamo? These issues are usually studied only in zonal averages by global helioseismology, but the Sun is longitudinally structured. Local helioseismology has revealed the presence of large-scale flows within the near-surface layers of the solar convection zone (e.g., Howe, 2008). These flows possess intricate patterns that change from one day to the next, accompanied by more gradually evolving patterns such as banded zonal flows and meridional circulation cells. These flow structures have been described as Solar Sub-surface Weather (SSW). Successive maps of these weather-like flow structures suggest that solar magnetism strongly modulates flow speeds and directions. Active regions tend to emerge in latitudes with stronger shear. The connections between SSW and active-region development are presently unknown.

2.2.1.1. Structure and Dynamics of the Tachocline Observation of the deep roots of solar activity in the tachocline is of primary importance for understanding the long-term variability of the Sun. HMI will use global and local helioseismic techniques to observe and investigate the large-scale character of the convection zone and tachocline. Tests with MDI 
observations and simulated data have shown that variations at the tachocline can be measured with local-helioseismology methods (Zhao et al., 2009). This and related helioseismology techniques will be used to study deep structures.

\subsubsection{Variations in Differential Rotation Differential rotation is a crucial component} of the solar cycle and is believed to generate the global-scale toroidal magnetic field seen in active regions. HMI will extend this useful data product with better near-surface resolution. Topics include solar differential rotation, relations between variations of rotation and magnetic fields, longitudinal variation of zonal flows ("torsional oscillations"), relations between the torsional pattern and active regions, sub-surface shear and its variations with solar activity, and the origin of the "extended" solar cycle (e.g., Howe et al., 2011).

\subsubsection{Evolution of Meridional Circulation Precise knowledge of meridional circulation} in the convection zone is crucial for understanding the long-term variability of the Sun. Helioseismology has found evidence for variation of the internal poleward flow during the solar cycle (e.g., Komm et al., 2011). To understand the global dynamics we must follow the evolution of the flow. HMI will enable measurement of the meridional circulation to significantly higher latitudes than has been possible with MDI and will generate continuous data for detailed, 3D maps of the evolving patterns of meridional circulation providing information about how flows transport and interact with magnetic fields throughout the solar cycle.

2.2.1.4. Dynamics in the Near-Surface Shear Layer Helioseismology has revealed that significant changes in solar structure over the solar cycle occur in the near-surface shear layer. However, the physics of these variations and their role in irradiance variations are still unknown. HMI will characterize the properties of this shear layer, the interaction between surface magnetism and evolving flow patterns, and the changes in structure and dynamics as the solar cycle advances. It will assess the statistical properties of convective turbulence over the solar cycle, including the kinetic helicity and its relation to magnetic helicity - two intrinsic characteristics of dynamo action.

\subsubsection{Origin and Evolution of Sunspots, Active Regions, and Complexes of Activity Ob-} servations show that magnetic flux on the Sun does not appear randomly. Once an active region emerges, there is a high probability that additional eruptions of flux will occur nearby (activity nests). How is magnetic flux created, concentrated, and transported to the solar surface where it emerges in the form of evolving active regions? To what extent are the appearances of active regions predictable? What roles do local flows play in their evolution? HMI will address these questions by providing tracked sub-surface sound-speed and flow maps for individual active regions and complexes under the visible surface of the Sun combined with surface magnetograms. Suggestions have been made that flux emerging in active regions originates in the tachocline. Flux is somehow ejected from the depths in the form of loops that rise through the convection zone and emerge through the surface. Phenomenological flux-transport models show that the observed photospheric distribution of the flux does not require a long-term connection to flux below the surface. Rather, field motions are described by the observed poleward flows, differential rotation, and surface diffusion acting on emerged flux of active regions. Does the active-region magnetic flux really disconnect from the deeper flux ropes after emergence? Are emerging flux regions really originate at the tachocline? 
2.2.1.6. Formation and Deep Structure of Magnetic Complexes of Activity HMI will allow exploration of the nature of long-lived complexes of solar activity, the principal sources of solar disturbances. "Activity nests" have been a puzzle for many decades. They may continue from one cycle to the next, and may be related to variations of solar activity on the scale of one to two years and short-term "impulses" of activity. HMI will probe beneath these features to $0.7 R_{\text {Sun }}$ the bottom of the convection zone, to search for correlated flow or thermal structures.

2.2.1.7. Active Region Source and Evolution By using acoustic tomography we can image sound-speed perturbations that accompany magnetic-flux emergence and disconnection that may occur. Vector magnetograms can give evidence on whether flux leaves the surface pre-dominantly as "bubbles", or whether it is principally the outcome of local annihilation of fields of opposing polarity. With a combination of helioseismic probing and vectorfield measurements, HMI will provide new insight into active-region flux emergence and removal.

2.2.1.8. Magnetic Flux Concentration in Sunspots Formation of sunspots is one of the long-standing questions of solar physics. Observations from MDI have revealed complicated flow patterns beneath sunspots and indicated that the highly concentrated magnetic flux in spots is accompanied by converging mass flows in the upper 3-4 Mm beneath the surface (Figure 3j). The evolution of these flows is not presently known. Detailed maps of subsurface flows in deeper layers, below $4 \mathrm{Mm}$, combined with surface fields and brightness for up to nine days during disk passage will allow investigation of the relations between flow dynamics and flux concentration in spots.

\subsubsection{Sources and Mechanisms of Solar Irradiance Variation Magnetic features -} sunspots, active regions, and network - that alter the temperature and composition of the solar atmosphere are primary sources of irradiance variability. How exactly do these features cause the irradiance variations? HMI together with SDO/AIA and SDO/EUV Variability Experiment (EVE: Woods et al., 2010) will study physical processes that govern these variations. The relation between interior processes, properties of magnetic field regions, and irradiance variations, particularly the UV and EUV components that have a direct and significant effect on Earth's atmosphere, will be studied.

\subsubsection{Sources and Drivers of Solar Activity and Disturbances It is commonly believed} that the principal driver of solar disturbances is stressed magnetic field. The stresses are released in the solar corona producing flares and coronal mass ejections (CME). The source of these stresses is believed to be in the solar interior. Flares usually occur in areas where the magnetic configuration is complex, with strong shears, high gradients, long and curved neutral lines, recent flux emergence, etc. This implies that the trigger mechanisms of flares are controlled by critical properties of magnetic field that lead eventually to MHD instabilities. But what kinds of instability actually govern, and under what conditions they are triggered, is unknown. With only some theoretical ideas and models, there is no certainty of how magnetic field is stressed or twisted inside the Sun or just what the triggering process is.

\subsubsection{Origin and Dynamics of Magnetic Sheared structures and $\alpha$-type Sunspots} Some spots contain two umbrae of opposite magnetic polarity within a common penumbra and are the source of powerful flares and CMEs. Such $\alpha$-type sunspot regions are thought 
to inject magnetic flux into the solar atmosphere in a highly twisted state. It is important to determine what processes beneath the surface lead to development of these spots and allow them to become flare and CME productive. This investigation will be carried out by analysis of evolving internal mass flows and magnetic-field topology of such spots.

\subsubsection{Magnetic Configuration and Mechanisms of Solar Flares Vector magnetic-field} measurements can be used to infer field topology and vertical electric current, both of which are believed to be essential to understand the flare process. Observations are required that can continuously track changes in magnetic field and electric current with sufficient spatial resolution to reveal changes of field strength and topology before and after flares. HMI will provide these unique measurements of the vector magnetic field over the whole solar disk with reasonable accuracy and at high cadence.

\subsubsection{Emergence of Magnetic Flux and Solar Transient Events Emergence of mag-} netic flux is closely related to solar transient events. MDI, GONG, and BBSO data show that there can be impulsive yet long-lived changes to the fields associated with eruptive events. The emergence of magnetic flux within active regions is often associated with flares. Emerging magnetic-flux regions near filaments lead to eruption of filaments. CMEs are also found to accompany emerging flux regions. Further, emergence of isolated active regions can proceed without any eruptive events. This suggests that magnetic flux emerging into the atmosphere interacts with pre-existing fields leading to loss of magnetic-field equilibrium. Observations of electric-current and magnetic topology differences between newly emerging and pre-existing fields will likely lead to the understanding of why emerging flux causes solar transient events. Vector polarimetry provided by HMI will enable these quantitative studies.

\subsubsection{Evolution of Small-Scale Structures and Magnetic Carpet The quiet Sun is cov-} ered with small regions of mixed polarity, sometimes termed "magnetic carpet", contributing to solar activity on short timescales. As these elements emerge through the photosphere they interact with each other and with larger magnetic structures. They may provide triggers for eruptive events, and their constant interactions may be a source of coronal heating. They may also contribute to irradiance variations in the form of enhanced network emission. While HMI will certainly not see all of this flux, it will allow global-scale observations of the small-scale element distribution, their interactions, and the resulting transformation of the large-scale field.

\subsubsection{Links Between the Internal Processes and Dynamics of the Corona and Heliosphere}

Intrinsic connectivity between multi-scale magnetic-field patterns increases coronal structure complexity leads to variability. For example, CMEs apparently interact with the globalscale magnetic field, but many CMEs, especially fast CMEs, are associated with flares, which are believed to be local phenomena. Model-based reconstruction of 3D magnetic structure is one way to estimate the field from observations. More realistic MHD coronal models based on HMI high-cadence vector-field maps as boundary conditions will greatly enhance our understanding of how the corona responds to evolving, non-potential active regions. 
2.2.2.1. Complexity and Energetics of the Solar Corona Observations from SOHO and TRACE have shown a variety of complex structures and eruptive events in the solar corona. However, categorizing complex structures has not revealed the underlying physics of the corona and coronal events. Two mechanisms have been proposed to generate stressed magnetic fields: photospheric shear motions and the emergence of already sheared magnetic fields; and both may, in fact, be at work on the Sun. But which plays the dominant role and how the energy injection is related to eruptive events are unknown. Magnetic helicity is an important characteristic of magnetic complexity and its conservation intrinsically links the generation, evolution, and reconnections of the magnetic field. HMI vector magnetic-field and velocity data (from both helioseismology and vector magnetic-field time-series-based velocity-inversion techniques) will allow better estimations of injections of energy and helicity into active regions. Observations from SDO/AIA and available coronagraphs ( $\mathrm{SOHO} /$ LASCO and STEREO) will show the subsequent response and propagation of complexity into the corona and heliosphere, relating the build-up of helicity and energy with energetic coronal events such as CMEs.

\subsubsection{Large-Scale Coronal Field Estimates Models computed from line-of-sight pho-} tospheric magnetic maps have been used to reproduce coronal forms that show multi-scale closed-field structures as well as the source of open field that starts from coronal holes but spreads to fill interplanetary space. Modeled coronal field demonstrates two types of closed field region: helmet streamers that form the heliospheric current sheet and a region sandwiched between the like-polarity open field regions (Zhao and Webb, 2003). There is evidence that most CMEs are associated with helmet streamers and with newly opened flux. HMI will provide uniform magnetic-field coverage at a high cadence, and together with simultaneous AIA and STEREO coronal images will enable the development of coronal magnetic-field models and study of the relationship between pre-existing patterns, newly opening fields, long-distance connectivity, and CMEs.

\subsubsection{Coronal Magnetic Structure and Solar Wind MHD simulation and current-free} coronal-field modeling based on magnetograms are two ways to study magnetic-field structuring of solar-wind properties. These methods have proven effective and promising, showing potential in applications of real-time space-weather forecasting. It has been demonstrated that modeling of the solar wind can be improved with increased cadence of the input magnetic data. By providing full-disk vector-field data at high cadence, HMI will enable these models to describe the distribution of the solar wind, coronal holes and open field regions, and how magnetic fields in active regions connect with interplanetary magnetic-field lines.

\subsubsection{Precursors of Solar Disturbances for Space-Weather Forecasts}

The number, strength, and timing of the strongest eruptive events are unpredictable at present. We are far from answering simple questions such as "will the next cycle be larger than the current one?" "When will the next large eruption occur?" Or even "when will there be several successive quiet days?" As we learn more about the fundamental processes through studies of internal motions, magnetic-flux transport and evolution, relations between active regions, UV irradiance, and solar-shape variations we will be vigilant for opportunities to develop prediction tools. Nevertheless, there are several near-term practical possibilities to improve the situation with HMI observations. 
2.2.3.1. Far-Side Imaging and Activity Index A procedure for solar far-side imaging was developed using data from MDI and has led to the routine mapping of the Sun's far side (Braun and Lindsey, 2001). Acoustic travel-time perturbations are correlated with strong magnetic fields, providing a view of active regions well before they become visible as they rotate onto the disk at the east limb. Synoptic images, which are now able to cover the entire far hemisphere of the Sun, will provide the ability to forecast the appearance on the visible disk of large active regions up to two weeks in advance and allow the detection of regions which emerge just a few days before rotating into view. HMI's full coverage to the limb will allow lower-noise far-side estimates.

\subsubsection{Predicting Emergence of Active Regions by Helioseismic Imaging Rising magne-} tic-flux tubes in the solar convection zone may produce detectable seismic signatures which would provide warning of their impending emergence. Helioseismic images of the base of the convection zone will employ a similar range of $p$ modes as those used to construct images of the far side. A goal is to detect and monitor seismic signatures of persistent or recurring solar activity near the tachocline. Success here could lead to long-term forecasts of solar activity.

\subsubsection{Determination of Magnetic Cloud $B_{S}$ Events Potentially valuable information for} geomagnetic forecasts - predictions of magnetic cloud $B_{\mathrm{S}}$ (southward field) events - can be obtained from the vector-field measurements. Long intervals of large southward interplanetary magnetic field and high solar-wind speed are believed to be the primary cause of intense geomagnetic disturbances, with the $B_{\mathrm{S}}$ component being the more important quantity. It has been shown that magnetic-field orientation in "clouds" remains basically unchanged while propagating from the solar surface to Earth's orbit. This provides a plausible chain of related phenomena that should allow prediction of the geo-effectiveness of CMEs directed toward Earth to be made from solar observations (see Siscoe and Schwenn, 2006, for a review). Estimates of embedded $B_{\mathrm{S}}$ should be significantly improved by incorporating frequently updated vector magnetic-field maps into coronal-field projections with the potential addition of coronagraphic observations from SOHO, SDO/AIA, and STEREO.

\section{Theoretical Support and Modeling}

The maximum scientific benefit from HMI can be obtained only with a specific theoretical program that includes numerical simulations of wave excitation and propagation in magnetized plasma, magneto-convection, local and global dynamo processes, magnetic-flux emergence, magnetic structures and MHD processes in the solar corona. Theoretical models for inversion of helioseismic and magnetic data are also extremely important for HMI data analyses. There are some key analysis capabilities that are not yet well understood. These include the interpretation of phases of acoustic waves measured in the presence of magnetic fields; the extraction of robust vector-field inferences with limited wavelength coverage; the problem of inferring coronal magnetic-field topology and properties (such as magnetic free energy) from the photospheric measurements, using force-free extrapolations, MHD models or other approaches; and understanding the helioseismic signals that might be detectable from sub-surface convection and fields. All of these areas are important to achieving the science goals of HMI. The inversion algorithms needs to be sufficiently fast to provide the scientific data products that are required for studying precursors of solar disturbances, such as full-disk maps of sub-surface flows, far-side images, and vector magnetograms, in almost 
real time. As a part of this project we will operate a 3D radiation MHD code that is being developed by HMI Co-Is at the NASA Ames Research Center. The members of the HMI research team will actively participate in the coordinated SDO Guest Investigator and LWS Theory Modeling and Data-Analysis Programs. It is clear that recent and ongoing developments in simulations and modeling will play an increasingly important role in progress for most HMI science goals.

\section{Data Products}

The HMI project has set the goal of producing a regular set of data products at a higher level of processing than has been the norm for most solar missions. In addition to the normal "Level-1 Science Data," which we interpret to be physical quantities such as Dopplergrams, magnetograms, continuum proxy, etc. which are already one stage beyond the observed filtergrams, we expect to provide standardized higher-level products such as sub-photospheric flow maps and coronal magnetic-field extrapolations. Such higher-level products have in the past been produced as byproducts of scientific analysis rather than a base product of the mission. These higher-level products will be based on the best available algorithms at the time that they are first put into production. The plan was to have the science team port the best existing code into the HMI processing pipeline late in the pre-launch phase to be ready early in the flight phase of the mission. This scenario counted on support to develop the techniques coming from outside the mission funding. In some cases this did not happen, so some of the planned products may not be available at the start. These data products are expected to evolve during the mission as the techniques of analysis continue to be developed.

There are some significant issues to be resolved before some of the products, e.g. robust inferences of sub-surface flows in active regions, can be provided.

Figure 3 shows samples of analyses that can be made using the planned standard data products.

The scientific data products can be divided into five main areas: global and local helioseismology, line-of-sight and vector magnetography, and continuum-intensity studies. These five primary scientific analyses cover all main HMI objectives, and have the following characteristics.

\subsection{Global Helioseismology}

The traditional normal-mode method will produce large-scale axisymmetrical distributions of sound speed, density, adiabatic exponent, and flow velocities through the whole solar interior from the energy-generating core to the near-surface convective boundary layer. These diagnostics will be based on frequencies and frequency splitting of modes of angular degree up to 1000 , obtained for several-day intervals as needed and up to $\ell=300$ for each 72-day interval. These will be used to produce a regular sequence of internal rotation and soundspeed inversions to allow observation from the tachocline to the near-surface shear layer. The techniques used here have been adapted from those used for MDI (Larson and Schou, 2011).

\subsection{Local-Area Helioseismology}

Local-area helioseismology methods such as time-distance techniques, ring-diagram analysis, and acoustic holography represent powerful tools for investigating physical processes 


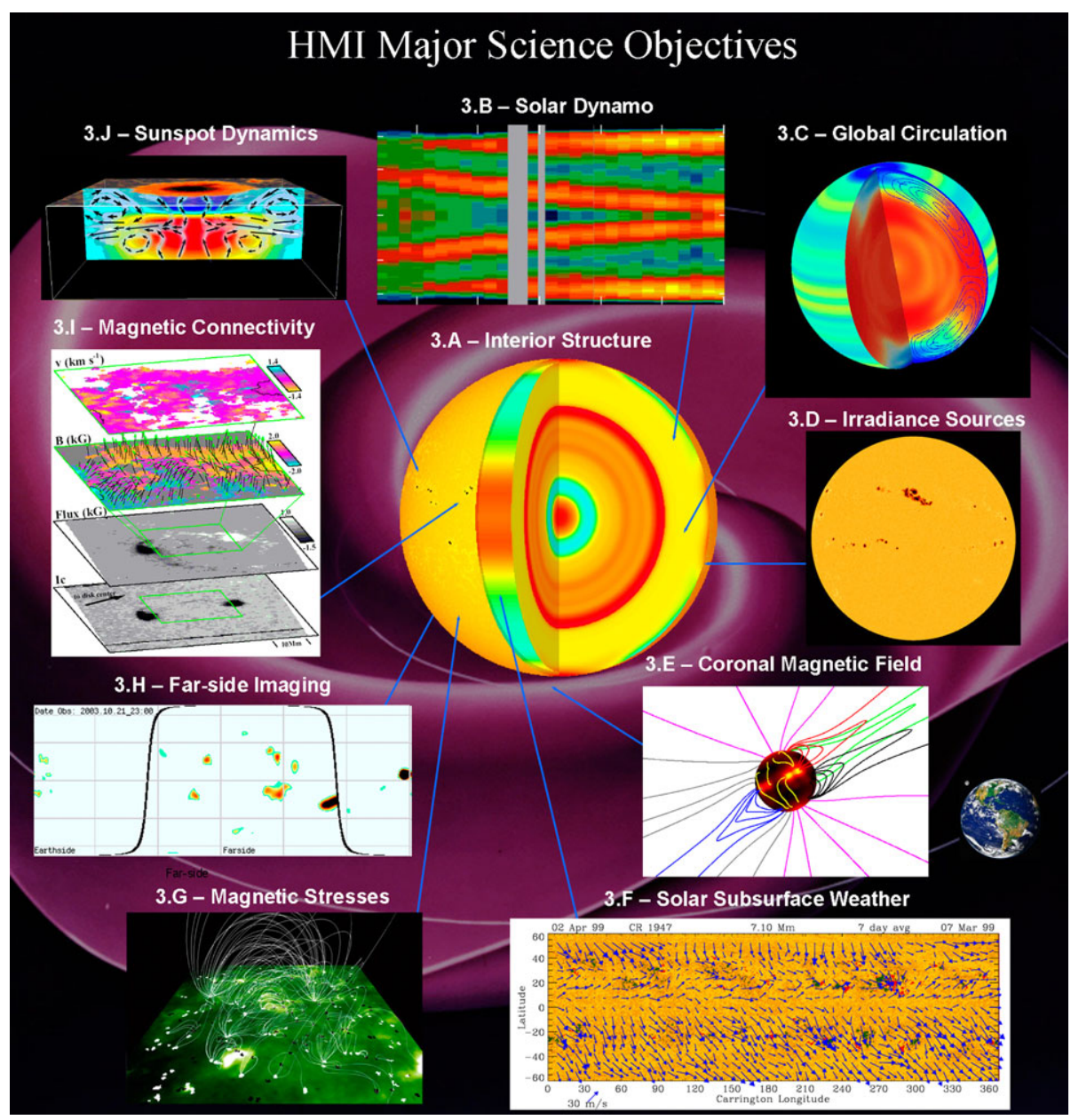

Figure 3 Samples of images that can be created using the planned HMI standard data products. The background is an artist's conception of the interplanetary current sheet as implied by extrapolated coronal fields (Wilcox, Hoeksema, and Scherrer, 1980). The overlays show: (A) Sound-speed variations relative to a standard solar model (Kosovichev, 2003); (B) Solar-cycle variations in the sub-photospheric rotation rate (Schou, private communication 2002); (C) Solar meridional circulation and differential rotation (http://soi.stanford.edu/press/ssu8-97/); (D) Sunspots and plage contribute to solar irradiance variation (SOHO/MDI); (E) MHD model of the magnetic structure of the corona (Linker and Mikic, 1997); (F) Synoptic map of the sub-surface flows at a depth of $7 \mathrm{Mm}$ (Haber et al., 2002); (G) EIT image and magneticfield lines computed from the photospheric field (Title, 1997); (H) Active regions on the far side of the Sun detected with helioseismology (SOHO/MDI); (I) Vector field image showing the magnetic connectivity in sunspots (Lites, Skumanich, and Martinez Pillet, 1998); (J) Sound-speed variations and flows in an emerging active region (Zhao, Kosovichev, and Duvall, 2001). This figure is from Scherrer and HMI Team (2002) with far-side image updated.

inside the Sun. These methods based on measuring local properties of acoustic and surfacegravity waves, such as travel times, frequency and phase shifts will provide images of internal structures and flows on various spatial and temporal scales and depth resolution. The targeted high-level regular data products include the following. 
4.2.0.4. Full-Disk Velocity and Sound-Speed Maps Maps of the upper convection zone (covering the top $20 \mathrm{Mm}$ ) obtained every eight hours with the time-distance methods on a Carrington grid of 120 by 120 degrees with a computational resolution of 0.12 degrees. These products are described by Zhao et al. (2011).

4.2.0.5. Synoptic Maps of Mass Flows and Sound-Speed Perturbations Synoptic maps are made for the upper convection zone for each Carrington rotation from averages of full-disk time-distance maps. These are made from day-wide North-South strips centered on the central meridian averaged from the eight-hour products described above.

4.2.0.6. Synoptic Maps of Horizontal Flows in Upper Convection Zone Maps are also made for each Carrington rotation with a 5-, 15-, and 30-degree resolution from ringdiagram analyses. These products are described by Bogart et al. (2011a, 2011b).

4.2.0.7. Higher-Resolution Maps Zoomed on Particular Active Regions Maps of sunspots and other targets, obtained with four- to eight-hour resolution for up to nine days continuously, with the time-distance method can be created on request as described in Zhao et al. (2011). Specific active regions or other regions of interest can also be processed through the "rings" pipeline upon request.

4.2.0.8. Deep-Focus Maps Maps spanning the whole convection zone depth, 0-200 Mm, with 10-15 degree resolution are planned but not yet developed to a data-product level of confidence.

4.2.0.9. Far-Side Images of the Sound-Speed Perturbations Maps associated with large active regions every 12 hours are computed using the GONG far-side pipeline.

In addition to this standard set of techniques, new approaches such as waveform tomography of small-scale structures will be deployed as they become developed.

The techniques for producing maps described above have been tested over several years with data from MDI and GONG and some with early HMI data. We are reasonably confident in inferences made in regions with little or no magnetic activity. However in regions of strong fields there are a number of issues that are either known to, or likely to, cause errors in inferences of physical parameters such as sound speed and flow velocities. Most of these issues are described by Kosovichev (2011) and references therein. Issues of phase variation with height as sampled are discussed by Rajaguru et al. (2010). Robust inferences of conditions in the vicinity of strong fields using local-area helioseismology can be achieved when these issues are properly accounted for in the analysis procedures.

\subsection{Magnetography}

The traditional line-of-sight component of the magnetic field is produced every 45 seconds as a co-product with the Doppler-velocity observations used for helioseismology. A 12minute average of the longitudinal field is also computed from the Stokes vector obtained as described in the next section. This observable has proven to be very useful in tracking magnetic-field evolution at all temporal and spatial scales. HMI will provide complete coverage of magnetic processes in the visible photosphere. Magnetograms will be assembled into synoptic and synchronic (similar to traditional Carrington maps but corrected for differential rotation) maps and extracted HMI active-region patches will be tracked across the Sun. Several products will be computed with various cadence and resolution for use as input to coronal magnetic-field and solar-wind models, activity forecasting, and correlative studies. 


\subsection{Vector Magnetic Field}

The vector field is one of the most-often absent important physical observables of the active solar atmosphere. HMI will produce several standard data series of vector fields. A 12-minute cadence full-disk analysis will be computed routinely and continuously to provide boundary conditions for large-scale coronal modeling. HMI, with the help of a robust inversion technique, will also provide AR-tracked and full-disk on-request vector magnetic field, and thermodynamic parameters of the photospheric plasma, with reasonable error estimates. These data will be used to quantitatively measure the free energy of the magnetic field, magnetic stresses, and helicity, providing important input to many prime science objectives and tasks of HMI and other SDO investigations. The HMI data products include the raw Stokes vector at six wavelengths across the line obtained each $90-135$ seconds and ordinarily averaged into a 12-minute product. Milne-Eddington inversions are routinely computed from these as described by Borrero et al. (2010). A further product is computed as the final vector field by applying a field direction disambiguation technique, since the Stokes parameters cannot distinguish the polarity of the component of the field normal to the line-of-sight (Metcalf, 1994; Metcalf et al., 2006; Leka et al., 2009). HMI provides a new challenge and opportunity for vector-field studies: observations are available for the full disk at all times. Thus while including all active regions with strong fields where the vector magnetic-field methods are expected to yield reasonably accurate measurements, the observations also include large areas of isolated fields where spatial averages may be useful but are hard to achieve in a robust manner. The optimization code for inference of non-linear force-free coronal magnetic fields is based on work by Wiegelmann (2004) and Wiegelmann, Inhester, and Sakurai (2006). We will also produce inferred velocities of the magnetic plasma, helicity, and Poynting fluxes based on tracking techniques (Welsch et al., 2007; Schuck, 2008).The magnetic data products are described in more detail at http://jsoc.stanford.edu/jsocwiki/MagneticField.

\subsection{Continuum Intensity}

The observations of an estimate of intensity in the continuum near the HMI spectral line will give a very useful measure of spot, faculae area, and other sources of irradiance. This will be important for studying the relationship between the MHD processes in the interior and lower atmosphere and irradiance variations. The continuum data will be also used for limb shape analysis, and for public information and education purposes. As side products of the Doppler and Continuum products HMI will produce line-depth and line-width estimates for the Fe I $617.3 \mathrm{~nm}$ line.

\subsection{Real-Time Products}

Data for observation planning for collaborative observing programs and for prediction of space-weather events are also produced as standard products. HMI Active Region Patches (HARPs) will be identified and tracked each 12 minutes. Line-of-sight magnetic field, continuum images, and other data products are available for space-weather investigations and forecasts in near-real time. Vector-field quantities can be computed with a slightly longer lag. Time series of computed space-weather quantities, such as total flux and neutral-line length, will be computed for each HARP on a rapid cadence. The near-real-time products are available with lags from the time of observation of less than 30 minutes. On a regular basis (daily or more often) we will provide full-disk maps of sound-speed distribution and mass flows in the upper convection zone, coronal magnetic field, predicted solar-wind velocity, and far-side activity-index maps. 


\section{Summary}

The HMI instrument is now on orbit as part of SDO and is producing data that appear to be of sufficient quality to address the goals described here. The JSOC data center is distributing data as needed. The data are available to the broad community and we hope it is productive to use HMI observations and data products to advance the broad goals of SDO.

Acknowledgements The HMI project is supported by NASA contract NAS5-02139. Efforts to develop science analysis code for the pipeline have been supported by NASA grants NNG05GM85G, NNG06GE40G, NNX07AP61G, NNX09AB10G, NNX09AG81G, and NNX10AC55G. We wish to thank Elizabeth Citrin the NASA SDO Program Manager, Barbara Thompson and Dean Pesnell the SDO project scientists, Madhulika Guhathakurta the NASA LWS program scientist, Arthur Poland, George Withbroe, and Richard Fisher at NASA Headquarters for both formative and continuing support.

The authors thank the entire HMI team for help in developing the investigation, instrument, and JSOC data system. In particular the partnership with the team at LMSAL was essential for the project to proceed. A project such as HMI is clearly a team effort with contributions from many. In addition to the authors, at least the following people contributed to the development of the HMI investigation and instrument (institutions as of launch): Hansen Experimental Physics Laboratory, Stanford University: Jim Aloise, Art Amezcua, John Beck (Co-I), Kelly Beck, Sudeepto Chakraborty, Millie Chethik, Keh-Cheng Chu, Carl Cimilluca, Sebastien Couvidat, Nancy Christensen, Romeo Durscher, Thomas Hartlep, Keiji Hayashi, Tim Huynh, Stathis Ilonidis, Kevin Kempter, Irina Kitiashvili, Rasmus Larsen, Tim Larson, Leyan Lo, Haruko Makatani, Rakesh Nigam, Konstantin Parchevsky, Bala Poduval, Brian Roberts, Kim Ross, Deborah Scherrer, Jeneen Sommers, Jennifer Spencer, Margie Stehle, Xudong Sun, Hao Thai, Karen Tian, Richard Wachter, Jeff Wade, XuePu Zhao (Co-I), and HEPL Staff, Lockheed Martin Solar and Astrophysics Laboratory: Dave Akin, Brett Allard, Ron Baraze, Mitch Baziuk, Tom Berger, E. Bogle, Bob Caravalho, Brock Carpenter, C. Cheung, Cathy Chou, Roger Chevalier, K. Chulick, Tom Cruz, Jerry Drake, Dexter Duncan, Jay Dusenbury, Janet Embrich, Chris Edwards, Cliff Evans, Peter Feher, Barbara Fischer, Charles Fischer, Samuel Freeland, Frank Friedlander, Glen Gradwohl, Henry Hancock, Gary Heyman, Bob Honeycutt, Elizabeth Hui, Bruce Imai, Jerry Janecka, Ramona Jimenez, Dwana Kacensky, Peter Kacensky, Claude Kam, Noah Katz, Karen Kao, Dave Kirkpatrick, Gary Kushner, Michael Levay, Russ Lindgren, Gary Linford, Andrea Lynch, Dnyanesh Mathur, Ed McFeaters, John Miles, Keith Mitchell, Sarah Mitchell, Ruth Mix, Margaret Morgan, Rose Navarro, Tom Nichols, Tracey Niles, Jackie Pokorny, Roger Rehse, Rick Rairden, John-Paul Riley, Lomita Rubio, David Schiff, Isela Scott, Ralph Sequin, Cheryl Seeley, Lawrence Shing, Araya Silpikul, Larry Springer, Richard Shine, Bob Stern, Louis Tavarez, Edgar Thomas, Ryan Timmons, Darrel Torgerson, Angel Vargas, Shan Varaitch, Dale Wolf, C. Jacob Wolfson, Carl Yanari, Ross Yamamoto, Kent Zickuhr, High Altitude Observatory: Juan Borrero Santiago, Gregory L. Card, Anthony Darnell, Rebecca C. Elliott, David Elmore, Jonathan Graham, Bruce Lites, Arturo Lopez Ariste, Matthias Rempel, Hector Socas-Navarro, Jet Propulsion Laboratory: Michael Turmon, JILA: Benjamin Brown, Gwen Dickenson, Nicholas Fetherstone, Deborah Haber, Bradley Hindman, Swati Routh, Regner Trampedach, Juri Toomre (Co-I), NASA Ames Research Center: Nagi Mansour (Co-I), Alan Wray (Co-I), New Jersey Institute of Technology/Big Bear Solar Observatory: Phil Goode (Co-I), Vasyl Yurchyshyn, North West Research Associates/Colorado Research Associates: Graham Barnes, Aaron Birch, Doug C. Braun (Co-I), Ashley Crouch, K.D. Leka (Co-I), Charles A. Lindsey (Co-I), Tom Metcalf (Co-I, deceased), Orion Poplawski, Martin Woodard, National Solar Observatory: Walter Allen, Olga Burtseva, Irene González Hernández, Frank Hill (Co-I), Rachel Howe (Co-I), Rudi Komm, Igor Suarez Sola, Sushanta Tripathy, Kiran Jain, Shakur Kholikov, Predictive Science Inc.: Michael Choy, Jon Linker (Co-I), Zoran Mickic, Pete Riley, Timofey Titov, Janvier Wijaya, Smithsonian Astrophysical Observatory: Alisdair Davey, Sylvain Korzennik (Co-I), University of California Los Angeles: Roger Ulrich (Co-I), University of Hawaii: Marcelo Emilio, Jeffrey R. Kuhn (Co-I), Isabelle Scholl, University of Maryland College Park: Judit Pap (Co-I), University of Southern California:Shawn Irish, Johann Reiter, Edward J. Rhodes Jr. (Co-I), Anthony Spinella, Yale University: Charles Baldner, Sarbani Basu (Co-I), Aarhus University: Jørgen Christensen-Dalsgaard (Co-I), Cambridge University: Douglas Gough (Co-I), European Space Agency: Bernhard Fleck (Co-I), Indian Institute of Astrophysics:Dipankar Banerjee, S. Paul Rajaguru, Siraj Hasan, James Cook University: Aimee Norton, Max Planck Institute for Solar System Research: Raymond Burston, Laurent Gizon, Yacine Saidi, Sami Solanki (Co-I), Mullard Space Science Laboratory: Elizabeth Auden, Len Culhane (Co-I), National Astronomical Observatory of Japan: Kaori Nagashima, Takashi Sekii (Co-I), Rutherford Appleton Laboratory: Richard Harrison (Co-I), Sarah Dunkin, Matthew Calpp, Nick Waltham, Sheffield University: Michael Thompson (Co-I), University of Tokyo: Hiromoto Shibahashi (Co-I), e2v: Gary Auker, Rob Wilson, Paul Jerram, LightMachinery: John Hunter, Ian Miller, Jeff Wimperis, Andover Corporation \& Zygo Corporation: John Cotton, H. Magnetics: Ralph Horber. 
Open Access This article is distributed under the terms of the Creative Commons Attribution Noncommercial License which permits any noncommercial use, distribution, and reproduction in any medium, provided the original author(s) and source are credited.

\section{References}

Bogart, R.S., Baldner, C., Basu, S., Haber, D.A., Rabello-Soares, M.C.: 2011a, HMI ring diagram analysis I. The processing pipeline. J. Phys. C 271(1), 012008. doi:10.1088/1742-6596/271/1/012008.

Bogart, R.S., Baldner, C., Basu, S., Haber, D.A., Rabello-Soares, M.C.: 2011b, HMI ring diagram analysis II. Data products. J. Phys. C 271(1), 012009. doi:10.1088/1742-6596/271/1/012009.

Borrero, J.M., Tomczyk, S., Kubo, M., Socas-Navarro, H., Schou, J., Couvidat, S., Bogart, R.: 2010, VFISV: Very fast inversion of the stokes vector for the helioseismic and magnetic imager. Solar Phys. doi:10.1007/s11207-010-9515-6.

Braun, D.C., Lindsey, C.: 2001, Seismic imaging of the far hemisphere of the Sun. Astrophys. J. Lett. 560, L189 - L192. doi:10.1086/324323.

Couvidat, S., Zhao, J., Birch, A.C., Kosovichev, A.G., Duvall, T.L., Parchevsky, K., Scherrer, P.H.: 2010, Implementation and comparison of acoustic travel-time measurement procedures for the solar dynamics observatory/helioseismic and magnetic imager time-distance helioseismology pipeline. Solar Phys. doi:10.1007/s11207-010-9652-y.

Couvidat, S., Schou, J., Shine, R.A., Bush, R.I., Miles, J.W., Scherrer, P.H., Rairden, R.L.: 2011, Wavelength dependence of the helioseismic and magnetic imager (HMI) instrument onboard the Solar Dynamics Observatory (SDO). Solar Phys. doi:10.1007/s11207-011-9723-8.

Drobnes, E., Littleton, A., Pesnell, W.D., Buhr, S., Beck, K., Durscher, R., Hill, S., McCaffrey, M., McKenzie, E., Scherrer, D., Wolt, A.: 2011, The SDO education and outreach (E/PO) program: Changing perceptions one program as a time. Solar Phys. accepted.

Duvall, T.L. Jr., Jefferies, S.M., Harvey, J.W., Pomerantz, M.A.: 1993, Time-distance helioseismology. Nature 362, 430 - 432. doi:10.1038/362430a0.

Duvall, T.L. Jr., Kosovichev, A.G., Scherrer, P.H., Bogart, R.S., Bush, R.I., de Forest, C., Hoeksema, J.T., Schou, J., Saba, J.L.R., Tarbell, T.D., Title, A.M., Wolfson, C.J., Milford, P.N.: 1997, Time-distance helioseismology with the MDI instrument: initial results. Solar Phys. 170, 63-73.

Haber, D.A., Hindman, B.W., Toomre, J., Bogart, R.S., Larsen, R.M., Hill, F.: 2002, Evolving submerged meridional circulation cells within the upper convection zone revealed by ring-diagram analysis. Astrophys. J. 570, 855 - 864. doi:10.1086/339631.

Hathaway, D., SDO SDT Panel: 2001, Solar Dynamics Observatory report of the science definition team. Technical report. Accessed 13 May 2011. http://www.nswp.gov/sdo/sdo_sdt_report.pdf.

Howe, R.: 2008, Helioseismology and the solar cycle. Adv. Space Res. 41, 846-854. doi:10.1016/j.asr.2006. 12.033.

Howe, R., Hill, F., Komm, R., Christensen-Dalsgaard, J., Larson, T.P., Schou, J., Thompson, M.J., Ulrich, R.: 2011, The torsional oscillation and the new solar cycle. J. Phys. C 271(1), 012074. doi:10.1088/1742$6596 / 271 / 1 / 012074$.

Komm, R., Howe, R., Hill, F., González Hernández, I., Haber, D.: 2011, Solar-cycle variation of zonal and meridional flow. J. Phys. C 271(1), 012077. doi:10.1088/1742-6596/271/1/012077.

Kosovichev, A.G.: 1996, Tomographic imaging of the Sun's interior. Astrophys. J. Lett. 461, L55-L57. doi:10.1086/309989.

Kosovichev, A.G.: 2003, What helioseismology teaches us about the Sun. In: Wilson, A. (ed.) Solar Variability as an Input to the Earth's Environment 535, ESA, Noordwijk, 795 - 806.

Kosovichev, A.G.: 2011, Local helioseismology of sunspots: Current status and perspectives. Solar Phys., submitted.

Kosovichev, A.G., HMI Science Team: 2004, HMI Science Plan. Accessed 13 May 2011. http://hmi.stanford. edu/doc/HMI-S014.pdf.

Kosovichev, A.G., Duvall, T.L. Jr., Scherrer, P.H.: 2000, Time-distance inversion methods and results (Invited review). Solar Phys. 192, 159- 176.

Larson, T., Schou, J.: 2011, HMI global helioseismology data analysis pipeline. J. Phys. C 271(1), 012062. doi:10.1088/1742-6596/271/1/012062.

Leka, K.D., Barnes, G., Crouch, A.D., Metcalf, T.R., Gary, G.A., Jing, J., Liu, Y.: 2009, Resolving the $180^{\circ}$ ambiguity in solar vector magnetic field data: Evaluating the effects of noise, spatial resolution, and method assumptions. Solar Phys. 260, 83 - 108. doi:10.1007/s11207-009-9440-8. 
Lemen, J.R., Title, A.M., Akin, D.J., Boerner, P.F., Chou, C., Drake, J.F., Duncan, D.W., Edwards, C.G., Friedlaender, F.M., Heyman, G.F., Hurlburt, N.E., Katz, N.L., Kushner, G.D., Levay, M., Lindgren, R.W., Mathur, D.P., McFeaters, E.L., Mitchell, S., Rehse, R.A., Schrijver, C.J., Springer, L.A., Stern, R.A., Tarbell, T.D., Wuelser, J.-P., Wolfson, C.J., Yanari, C., Bookbinder, J.A., Cheimets, P.N., Caldwell, D., Deluca, E.E., Gates, R., Golub, L., Park, S., Podgorski, W.A., Bush, R.I., Scherrer, P.H., Gummin, M.A., Smith, P., Auker, G., Jerram, P., Pool, P., Soufli, R., Windt, D.L., Beardsley, S., Clapp, M., Lang, J., Waltham, N.: 2011, The atmospheric imaging assembly (AIA) on the Solar Dynamics Observatory (SDO). Solar Phys. doi:10.1007/s11207-011-9776-8.

Linker, J.A., Mikic, Z.: 1997, Extending coronal models to Earth orbit. In: Crooker, N., Joselyn, J., Feynman, J. (eds.) Coronal Mass Ejections: Causes and Consequences, Geophys. Monogr. Ser. 99, AGU, Washington.

Lites, B.W., Skumanich, A., Martinez Pillet, V.: 1998, Vector magnetic fields of emerging solar flux. I. Properties at the site of emergence. Astron. Astrophys. 333, 1053-1068.

Mason, G., LWS Panel: 2001, Living with a star science architecture team report to SECAS. Technical report. Accessed 13 May 2011. http://www.nswp.gov/lwsgeospace/SECAS/LWSSAT_SECASreport _30Aug01.pdf.

Metcalf, T.R.: 1994, Resolving the 180-degree ambiguity in vector magnetic field measurements: The 'minimum' energy solution. Solar Phys. 155, 235 - 242. doi:10.1007/BF00680593.

Metcalf, T.R., Leka, K.D., Barnes, G., Lites, B.W., Georgoulis, M.K., Pevtsov, A.A., Balasubramaniam, K.S., Gary, G.A., Jing, J., Li, J., Liu, Y., Wang, H.N., Abramenko, V., Yurchyshyn, V., Moon, Y.-J.: 2006, An overview of existing algorithms for resolving the $180^{\circ}$ ambiguity in vector magnetic fields: Quantitative tests with synthetic data. Solar Phys. 237, 267 - 296. doi:10.1007/s11207-006-0170-x.

Rajaguru, S.P., Wachter, R., Sankarasubramanian, K., Couvidat, S.: 2010, Local helioseismic and spectroscopic analyses of interactions between acoustic waves and a sunspot. Astrophys. J. Lett. 721, L86-L91. doi:10.1088/2041-8205/721/2/L86.

Scherrer, P.H., HMI Team: 2002, Helioseismic and magnetic imager for Solar Dynamics Observatory. Accessed 13 May 2011. http://hmi.stanford.edu/doc/HMI-S001.pdf.

Scherrer, P.H., Bogart, R.S., Bush, R.I., Hoeksema, J.T., Kosovichev, A.G., Schou, J., Rosenberg, W., Springer, L., Tarbell, T.D., Title, A., Wolfson, C.J., Zayer, I., MDI Engineering Team: 1995, The solar oscillations investigation - Michelson Doppler Imager. Solar Phys. 162, 129-188. doi:10.1007/ BF00733429.

Schou, J., Antia, H.M., Basu, S., Bogart, R.S., Bush, R.I., Chitre, S.M., Christensen-Dalsgaard, J., di Mauro, M.P., Dziembowski, W.A., Eff-Darwich, A., Gough, D.O., Haber, D.A., Hoeksema, J.T., Howe, R., Korzennik, S.G., Kosovichev, A.G., Larsen, R.M., Pijpers, F.P., Scherrer, P.H., Sekii, T., Tarbell, T.D., Title, A.M., Thompson, M.J., Toomre, J.: 1998, Helioseismic studies of differential rotation in the solar envelope by the solar oscillations investigation using the Michelson Doppler Imager. Astrophys. J. 505, 390 - 417. doi:10.1086/306146.

Schou, J., Borrero, J.M., Norton, A.A., Tomczyk, S., Elmore, D., Card, G.L.: 2010, Polarization calibration of the helioseismic and magnetic imager (HMI) onboard the Solar Dynamics Observatory (SDO). Solar Phys. doi:10.1007/s11207-010-9639-8.

Schou, J., Scherrer, P.H., Bush, R.I., Wachter, R., Couvidat, S., Rabello-Soares, M.C., Bogart, R.S., Hoeksema, H.T., Liu, T., Duvall, J.T.L., Akin, D.J., Allard, B.A., Miles, J.W., Rairden, R., Shine, R.A., Tarbell, T.D., Title, A.M., Wolfson, C.J., Elmore, D.F., Norton, A.A., Tomczyk, S.: 2011, The helioseismic and magnetic imager instrument design and ground calibration. Solar Phys. doi:10.1007/s11207-011-9842-2.

Schuck, P.W.: 2008, Tracking vector magnetograms with the magnetic induction equation. Astrophys. J. 683, 1134 - 1152. doi:10.1086/589434.

Siscoe, G., Schwenn, R.: 2006, CME disturbance forecasting. Space Sci. Rev. 123, 453-470. doi:10.1007/ s11214-006-9024-y.

Title, A.M.: 1997, Solar mystery near solution with data from SOHO spacecraft, the Sun's newly-discovered magnetic carpet may explain coronal heating. Accessed 13 May 2011. http://soi.stanford.edu/press/ ssu11-97/.

Title, A.M., Schrijver, C.J.: 1998, The Sun's magnetic carpet. In: Donahue, R.A., Bookbinder, J.A. (eds.) Cool Stars, Stellar Systems, and the Sun 154, Astron. Soc. Pac., San Francisco, 345.

van Driel-Gesztelyi, L., Culhane, J.L.: 2009, Magnetic flux emergence, activity, eruptions and magnetic clouds: Following magnetic field from the Sun to the heliosphere. Space Sci. Rev. 144, $351-381$. doi:10.1007/s11214-008-9461-x.

Wachter, R., Schou, J., Rabello-Soares, M.C., Miles, J.W., Duvall, T.L., Bush, R.I.: 2011, Image quality of the helioseismic and magnetic imager (HMI) onboard the solar dynamics observatory (SDO). Solar Phys. doi:10.1007/s11207-011-9709-6. 
Welsch, B.T., Abbett, W.P., De Rosa, M.L., Fisher, G.H., Georgoulis, M.K., Kusano, K., Longcope, D.W., Ravindra, B., Schuck, P.W.: 2007, Tests and comparisons of velocity-inversion techniques. Astrophys. J. 670, 1434 - 1452. doi: $10.1086 / 522422$.

Wiegelmann, T.: 2004, Optimization code with weighting function for the reconstruction of coronal magnetic fields. Solar Phys. 219, 87 - 108. doi:10.1023/B:SOLA.0000021799.39465.36.

Wiegelmann, T., Inhester, B., Sakurai, T.: 2006, Preprocessing of vector magnetograph data for a nonlinear force-free magnetic field reconstruction. Solar Phys. 233, 215-232. doi:10.1007/s11207-006-2092-z.

Wilcox, J.M., Hoeksema, J.T., Scherrer, P.H.: 1980, Origin of the warped heliospheric current sheet. Science 209, 603 - 605. doi:10.1126/science.209.4456.603.

Woods, T.N., Eparvier, F.G., Hock, R., Jones, A.R., Woodraska, D., Judge, D., Didkovsky, L., Lean, J., Mariska, J., Warren, H., McMullin, D., Chamberlin, P., Berthiaume, G., Bailey, S., Fuller-Rowell, T., Sojka, J., Tobiska, W.K., Viereck, R.: 2010, Extreme ultraviolet variability experiment (EVE) on the Solar Dynamics Observatory (SDO): Overview of science objectives, instrument design, data products, and model developments. Solar Phys. doi:10.1007/s11207-009-9487-6.

Zhao, J., Kosovichev, A.G., Duvall, T.L. Jr.: 2001, Investigation of mass flows beneath a sunspot by timedistance helioseismology. Astrophys. J. 557, 384 - 388. doi:10.1086/321491.

Zhao, J., Hartlep, T., Kosovichev, A.G., Mansour, N.N.: 2009, Imaging the solar tachocline by time-distance helioseismology. Astrophys. J. 702, 1150-1156. doi:10.1088/0004-637X/702/2/1150.

Zhao, J., Couvidat, S., Bogart, R.S., Parchevsky, K.V., Birch, A.C., Duvall, T.L., Beck, J.G., Kosovichev, A.G., Scherrer, P.H.: 2011, Time-distance helioseismology data-analysis pipeline for helioseismic and magnetic imager onboard Solar Dynamics Observatory (SDO/HMI) and its initial results. Solar Phys. doi:10.1007/s11207-011-9757-y.

Zhao, X.P., Webb, D.F.: 2003, Source regions and storm effectiveness of frontside full halo coronal mass ejections. J. Geophys. Res. 108, 1234. doi:10.1029/2002JA009606.

Zwaan, C.: 1987, Elements and patterns in the solar magnetic field. Annu. Rev. Astron. Astrophys. 25, $83-$ 111. doi:10.1146/annurev.aa.25.090187.000503. 